\title{
Development and Analysis of a New Cylindrical Lithium-ion Battery Thermal Management System
}

Yasong Sun ( $\nabla$ yssun@nwpu.edu.cn )

Northwestern Polytechnical University https://orcid.org/0000-0002-2519-2788

Ruihuai Bai

Northwestern Polytechnical University

Original Article

Keywords: Cooling system, Electric vehicle, Kriging approximation, Numerical simulation

Posted Date: November 23rd, 2021

DOI: https://doi.org/10.21203/rs.3.rs-934197/v2

License: (c) (i) This work is licensed under a Creative Commons Attribution 4.0 International License. Read Full License 


\section{Cover letter}

October 20, 2021

\section{Dear Editor}

We are sending you a new paper titled as "Development and analysis of a new cylindrical lithium-ion battery thermal management system" by Yasong Sun, and Ruihuai Bai to Chinese Journal of Mechanical Engineering for consideration.

The paper is submitted as an invited paper of EVS34/ICEIV2021 conferences. In the EVS34/ICEIV2021, the paper ID and title are "14001441" and "Development and analysis of a new cylindrical lithium-ion battery thermal management system", respectively.

The new paper has been rewritten, and the Overall Similarity Index Percentage is less than $12 \%$. In the present paper, our own original works and others worldwide are reviewed and commented. Perspective research directions are proposed. We believe that the new paper will attract broad interest to readers in energy society.

The new submission satisfies the journal requirement and is well written in English. The paper has not been published previously and will not be submitted elsewhere before we receive your decision. We appreciate your handling of the paper.

Sincerely yours,

Yasong Sun, Ruihuai Bai 


\section{Title page}

\section{Development and analysis of a new cylindrical lithium-ion battery thermal management system}

Ya-Song Sun, born in 1986, is currently an associate professor at School of Power and Energy, Northwestern Polytechnical University, China. He received his $\mathrm{PhD}$ degree from Northwestern University, China, in 2011. His research interests focus on heat and mass transfer in power battery.

Tel: +86-029-88431112; E-mail: yssun@nwpu.edu.cn

Rui-Huai Bai, born in 1997, is currently a master candidate at School of Power and Energy, Northwestern Polytechnical University, China. E-mail: 598295322@qq.com

Corresponding author: Ya-song Sun E-mail: yssun@nwpu.edu.cn 


\title{
Development and analysis of a new cylindrical lithium-ion battery thermal management system
}

\author{
Ya-Song Sun ${ }^{1,2} \cdot$ Rui-Huai Bai ${ }^{2}$
}

Received September 28, 2021; revised February xx, 202x; accepted March xx, 202x

(C) Chinese Mechanical Engineering Society and Springer-Verlag Berlin Heidelberg 2021

\begin{abstract}
'Abstract: With the development of modern technology and the economy, environmental protection and sustainable development have become the focus of global attention. In this paper, the promotion and development of electric vehicles have bright prospects, and they are also facing many challenges. Under different operating conditions, various safety problems of electric vehicles emerge one after another, especially the potential safety hazards caused by battery overheating that threaten electric vehicles' development process. In this paper, a new indirect liquid cooling system is designed and optimized for cylindrical lithiumion batteries. A variety of design schemes for different cooling channel structures and cooling liquid inlet direction are proposed, and the corresponding solid-fluid coupling model is established. COMSOL Multiphysics simulation software models, simulates and analyses cooling systems. An approximate model is constructed using the Kriging method, and it is considered to optimize the battery cooling system and improve the optimization results. Sensitivity parameter analysis and system structure optimization design are also carried out on the influencing factors of the battery thermal management. The results indicate it effectively balances and reduces the maximum core temperature and temperature difference of the battery pack. Compared with the original design, from the optimized design of these factors, which based on method of the non-dominated sorting genetic algorithm (NSGA-II), there is an excellent ability on the optimized thermal management system to dissipate thermal energy and keep the overall cooling uniformity of the battery and thermal management system. Furthermore, under thermal abuse conditions, the optimized system can also prevent thermal runaway propagation. In summary, this research is expected to provide some practical

Ya-Song Sun

yssun@nwpu.edu.cn

1 School of Power and Energy, Northwestern Polytechnical University, Xi'an 710072, China
\end{abstract}

suggestions and ideas for the engineering and production applications and structural optimization design carried by electric vehicles.

Keywords: Cooling system • Electric vehicle • Kriging approximation $\bullet$ Numerical simulation

\section{Introduction}

With the increasing of serious environmental pollution, the concept of environmental protection is constantly deepening on a global scale. To reduce urban carbon emissions and improve air quality, the promotion and development of electric vehicles are of great significance. As the power source of electric vehicles, lithium batteries have high specific energy and long cycle life advantages. However, due to the characteristics of being sensitive to temperature changes [1], and lots of battery thermal runaway problems occur under extreme ambient temperatures. And, battery thermal management (BTM) is the key to solving a series of issues such as battery heat dissipation in electric vehicles [2].

At present, the BTM system used in electric vehicles can be divided into three forms: air cooling, liquid cooling, and phase change cooling [3]. The air-cooling type has some great abilities of more choices of pipeline layout, high-cost performance, and lower maintenance costs [4]. However, due to the limited heat dissipation capacity of the air-cooled BTM system, poor cooling uniformity, and low NVH (noise, vibration, and roughness) [5], its application has been limited. The Phase change material (PCM) BTM system is a reliable cooling technology with advantages such as

2 Center of Computational Physics and Energy Science, Yangtze River Delta Research Institute of NPU, Northwestern Polytechnical University, Taicang, Jiangsu, 215400, China 
reliability and safety of lithium batteries [6]. However, this system is still in the laboratory research and exploration stage and has not yet been widely accepted and recognized [7]. BTM systems mainly include pure PCM, composite PCM, and hybrid PCM. Chen et al. [8] review the advantages and disadvantages of BTM systems-based PCM. They got conclusions through research that thermal conductivity in most of the single PCM systems is very low, which results in a significant amount of thermal energy accumulation in the battery system in an extreme temperature working environment. To improve the thermal conductivity and strength of the phase change material, carbon materials and metals can be optionally added. The hybrid BTM system has a better effect on improving the uniformity of battery temperature distribution and can further reduce battery temperature rise. In addition, the heat dissipation problem of the battery module is studied based on the orthogonal experimental design [9]. In addition, they also studied the application of graphene in energy storage technology and thermal energy transfer. Due to the superior cooling performance and more flexible pipeline layout, the liquid cooling strategy has been widely applied in electric vehicles, such as Tesla Model S and Chevrolet Volt. The thermal management of a cylindrical battery with double profiles is more complicated than a six-sided cylindrical unit. As the power density of the battery increases, the thermal energy produced in the cylindrical battery also increases. Therefore, the liquid-cooled BTM system has become an essential and applicable solution for lithium battery cooling technology in electric vehicles [11]. Tesla Motors [11] designed a bellows belt with a double inlet and outlet to control the heat distribution of the 18650 battery. Zhao et al. [12] developed and researched a cooling jacket with microchannels to control the temperature distribution of the 18650 battery. They also investigated the effects of channel number, channel size and fluid flow on the temperature profile of the battery. Rao et al. [13] used a heat-conducting device with a liquid channel to cool the cylindrical battery. This device can effectively dissipate the heat generated by battery. Sheng et al. [14] developed a battery liquid cooling jacket that satisfies the requirements of its cooling effect for the 21700 lithium-ion battery. They numerically investigated the influence of fluid flow, channel size and cooling medium on the thermal characteristics of the battery. These numerical results have been validated by experiment.

To simplify the evaluation and simulation of battery performance, Hallaj et al. [15] developed a one-dimensional mathematical model to simulate the internal temperature curve of cylindrical lithium-ion batteries, and analyzed the effect of simplified batteries. Because the BTM system was a high cooling rate, the sensitivity of the electrochemical reaction of the cylindrical battery to the temperature gradient can be ignored. Al-Zareer et al. [16] proposed a one-dimensional model to simulate the electrochemical reaction, and a three-dimensional model to simulate the thermal distribution of the battery. Since the purpose of the BTM system is to stabilize the temperature in the best working section, modelling the electrochemical reaction at the average temperature of the battery can accurately predict the amount of heat generated.

In this paper, a new type of liquid cooling BTM system is proposed to solve poor temperature rise and non-uniform distribution of 21700 cylindrical batteries. To further improve the heat dissipation effect of the battery, add a cooling channel in the center of adjacent batteries and increase the diameter of the cooling channel. The effects of the flow direction of the cooling medium in the flow channel and the structure size of the cooling channel on the cooling performance of the BTM system is analyzed, and the BTM system was optimized with numerical simulation. This investigation can effectively guide the design of cylindrical battery.

\section{Physical and numerical models}

\subsection{Physical model}

The integrated battery pack BTM system [17] is shown in Figure 1. The integrated battery pack is composed of multiple 21700 batteries in parallel and embedded in the aluminium cooling sleeve. The liquid cooling holes are distributed between the batteries, and the cooling medium (water) flows through the cooling channel to cool the cooling sleeve and take away the thermal energy generated by the battery. The physical model of the battery pack can be simplified as the right hand of Figure 1 using symmetries.

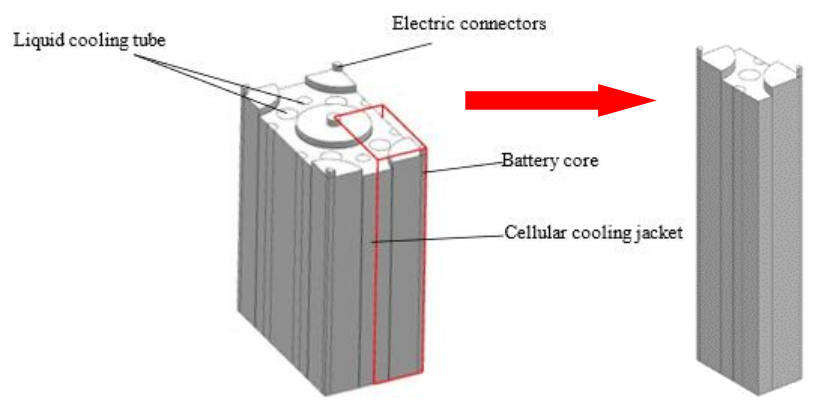

Figure 1 The battery pack and simplified model

As shown in Figure 1, the battery model is established by the actual parameters of the 21700 battery. The height and outer diameters are $70 \mathrm{~mm}$ and $10.72 \mathrm{~mm}$, respectively. The core diameter and shell thickness of the battery are $3.2 \mathrm{~mm}$ 
and $0.22 \mathrm{~mm}$, respectively.

The COMSOL Multiphysics software [18] is used to model, simulate and analyze the BTM system. It is a comprehensive multi-platform finite element solver that can simulate electronic, physical and mechanical systems.

\subsection{Numerical model}

In order to study the cooling performance of BTM system and obtain the distribution of battery temperature, two independent models are developed to simulate the integrated battery pack and BTM system. One model is a onedimensional electrochemical model, and this model is constructed to simulate the electrochemical reaction of the battery and the temperature variation under different working conditions. The other model is a three-dimensional thermal energy transfer model which composed of the battery pack and BTM system.

Since the electrochemical reaction in the spiral direction can be ignored, the electrochemical performance of the cylindrical battery can be evaluated by one-dimensional electrochemical model [19]. The simulation domain and boundary conditions of this model are shown in Figure 2.

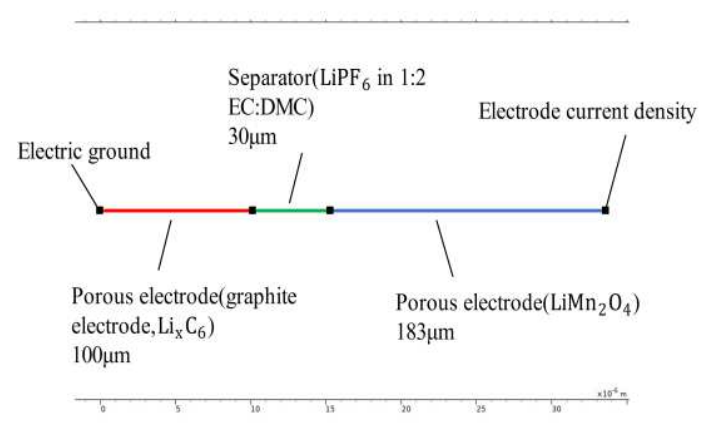

Figure 2 The computational domain of electrochemical model and boundary condition

The electrochemical model and thermal energy transfer model are coupled by the generated heat source and average temperature. These two models jointly determine the cooling performance of the proposed BTM system. The onedimensional electrochemical model obtains the average temperature of the battery from the thermal energy transfer model, and further feeds the average heat source back to the thermal energy transfer model through simulation calculation. The heat and temperature distribution of the battery is further simulated and calculated by the Thermal energy transfer model.

\subsubsection{Three-dimensional thermal energy transfer model}

The computational domain of the three-dimensional thermal energy transfer model is shown in Figure 3(a). Two-quarters of batteries and cooling channels in the domain are embedded in the aluminium cooling sleeve. The mesh quality selected for the three-dimensional thermal energy transfer model is shown in Figure 3(b). The control equations can be expressed by the energy balance equation, the mass, momentum and energy conservation equations of the coolant. The conservation equation of battery energy rate can be expressed as

$$
\frac{\partial}{\partial t}\left(\rho_{b} c_{p, b} T_{b}\right)=\nabla \cdot\left(k_{b} \nabla T_{b}\right)+\dot{Q}_{g e n}
$$

where $\rho_{b}, c_{p, b}$ and $k_{b}$ are the battery density, specific heat capacity and thermal conductivity of battery material, respectively. $\nabla T_{b}$ represents the temperature distribution of the battery in a process, $\dot{Q}_{g e n}$ is the thermal energy generated by the battery.


Figure 3 (a) The computational domain of three-dimensional thermal energy transfer model (b) The quality of mesh

The mass, momentum and energy balance equation of the coolant are

$$
\begin{gathered}
\frac{\partial \rho_{b}}{\partial t}+\nabla \cdot\left(\rho_{P} \vec{v}\right)=0 \\
\frac{\partial}{\partial t}\left(\rho_{P} \vec{v}\right)+\nabla \cdot\left(\rho_{P} \vec{v} \vec{v}\right)=-\nabla P+\rho g \\
\frac{\partial}{\partial t}\left(\rho_{b} c_{p, b} T_{b}\right)+\nabla \cdot\left(\rho_{b} c_{p, b} \vec{v} T_{p}\right)=\nabla \cdot\left(k_{p} \nabla T_{p}\right)
\end{gathered}
$$

where the subscript $p$ is the cooling medium, $P$ is the pressure, $g$ is the gravity acceleration, $\vec{v}$ is the velocity vector of coolant.

To obtain the volume heating rate of the electrochemical model and apply it to the active material part of the battery model. Compared with the heat production rate of the BTM system, the heat production rate at the battery connector can be ignored. Thus, the insulation boundary condition is used. Assuming that the active battery material consists of one or 
more single cells, these single cells spirally wound to form a columnar. Therefore, the thermal conductivity in the thermal energy transfer model is anisotropic, and the thermal conductivity along the direction of the battery plate (cylinder length direction) is higher than that in the normal (radial) direction of the battery plate.

In the radial direction, the thermal conductivity $k_{r}$ is calculated by the following formula [20]

$$
k_{r}=\frac{\Sigma L_{i}}{\Sigma\left(\frac{L_{i}}{k_{i}}\right)}
$$

where $L_{i}$ is the thickness of different layers of a single battery, and $k_{i}$ represents the thermal conductivity of the materials.

The thermal conductivity in the length direction of cylinder is

$$
\mathrm{k}_{\mathrm{z}}=\frac{\sum\left(L_{\mathrm{i}} \mathrm{k}_{\mathrm{i}}\right)}{\Sigma L_{\mathrm{i}}}
$$

Similarly, $\rho_{b}$ and $c_{p, b}$ are density and heat capacity of battery materials

$$
\begin{gathered}
c_{p, b}=\frac{\sum\left(L_{i} c_{p, i}\right)}{\sum\left(L_{i}\right)} \\
c_{p, b}=\frac{\sum\left(L_{i} \rho_{i}\right)}{\sum\left(L_{i}\right)}
\end{gathered}
$$

\subsubsection{One-dimensional electrochemical model}

The one-dimensional electrochemical model was established by Doyle et al. [21] and Fuller et al. [22]. The constant current charge and discharge of the lithium anode battery were modeled by using the concentrated solution theory. The equilibrium voltage changes of negative and positive electrode materials are based on the experimental results of Doyle et al. [19]. Fuller et al. [22] further studied the double lithium-ion insertion battery and optimized the active battery material.

The two porous electrodes sandwiched in the third layer of non-aqueous electrolyte are the main structure of the battery. The electrode has a porous structure, and the diaphragm is a multi-component system of plastic composed of five polymers, two kinds of liquids, one cation, and one anion. The following relationship can describe the change of the salt concentration in the electrode solution

$$
\frac{\partial b_{s}}{\partial t}=\frac{1}{\varepsilon}\left(\nabla \cdot\left(D_{s} \nabla b_{s}\right)+\left(a j_{n}+a j_{n} t_{+}^{0}\right)-\frac{i_{2} \cdot \nabla t_{+}^{0}}{F}\right)
$$

in the above formula, $b_{s}$ represents the salt concentration in the electrode, $D_{S}$ represents diffusion coefficient of the electrode, $a$ represents particular interface part, $t$ represents time, $i_{2}$ represents current density at the surface of the liquid phase, and $j_{n}$ represents lithium-ion pore wall flux. The subscript "+" in the formula represents positive and "-." represents negative. The relationship between lithium-ion surface current density and lithium-ion pore wall flux is

$$
j_{n}(a F)=\nabla \cdot i_{2}
$$

where the surface density of the liquid phase $i_{2}$ represents a function of electrolyte concentration, time, conductivity, absolute temperature, activity coefficient, and electrode solution phase potential. It can be expressed as

$$
\begin{gathered}
i_{2}=-k \nabla \phi_{2}+\left(\frac{2 k R T}{F}+\frac{2 k R T}{F} \cdot \frac{\partial \ln f_{ \pm}}{\partial \ln b_{S}}\right) \\
\left(1-t_{+}^{0}\right) \nabla \ln b_{S}
\end{gathered}
$$

$F$ represents Faraday constant that has value of 96,587 $\mathrm{C} / \mathrm{mol}$ and $R$ represents general gas constant. The solid phase current density $\left(i_{1}\right)$ is the function of solid-electrode potential and electronic conductivity of solid phase mixture,

$$
i_{1}=-\sigma \nabla \phi_{1}
$$

The concentration of lithium element in the solid matrix of the battery electrode can be expressed by the following formula

$$
\frac{\partial b_{L i}}{\partial t}=D_{L i}\left[\frac{2}{r} \frac{\partial b_{L i}}{\partial r}+\frac{\partial^{2} b_{L i}}{\partial r^{2}}\right]
$$

where $D_{L i}$ represents diffusion coefficient of lithium ion in electrode solid phase, $r$ is radial direction of battery.

The following formula is the Butler-Volmer kinetic equation, which connect the solid and liquid phase.

$$
\begin{gathered}
\left(\frac{j_{n}}{H}\right)+\left(b_{S}\right)^{0.5}\left(b_{i}-b_{L i}\right)^{0.5}\left(b_{L i}\right)^{0.5} \exp \left(-\frac{F}{2 R T}\left(\eta-V_{o c}\right)\right)= \\
\left(b_{S}\right)^{0.5}\left(b_{i}-b_{L i}\right)^{0.5}\left(b_{L i}\right)^{0.5} \exp \left(\frac{F}{2 R T}\left(\eta-V_{o c}\right)\right)
\end{gathered}
$$




$$
\frac{\partial b_{s}}{\partial r}=-j_{n} / D_{L i}
$$

$H$ represents the result of multiplying positive and negative chemical reaction constants, $b_{i}$ represents the salt concentration of the $i$ th layer, $b_{L i}$ represents the solid lithium concentration, the battery electrode potential is represented by $\eta, V_{o c}$ represents value of open circuit voltage. The battery electrode potential is expressed by the following formula

$$
\eta=\phi_{1}-\phi_{2}
$$

As shown in Figure 4, the curves of potential and battery capacity of the one-dimensional electrochemical model at different rates were compared with the research conclusion of Doyle et al. [21]. The comparison results show that the model can accurately predict the electrochemical behavior of lithium-ion batteries.

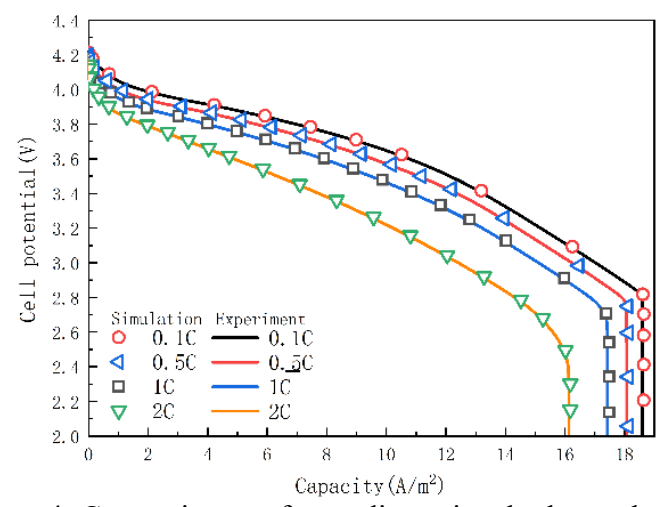

Figure 4 Comparisons of one-dimensional electrochemical model at different rates with Doyle et al. [18].

\section{The effects of flow direction and cooling channel sizes}

In this section, the effects of flow direction of the cooling medium and structure size of the cooling channel on the cooling performance of BTM system are analyzed. The initial temperature and ambient temperature are set to 303 $\mathrm{K}$, and the average temperature change of the battery core shaft was monitored in the simulation process. The battery temperature rise is assumed as $5 \mathrm{~K}$.

\subsection{The effect of flow direction}

This section mainly analyzes the influence of fluid flow direction on the cooling performance of initial designed BTM system. As shown in Figure 5, according to the different positions of the inlet and outlet of the cooling channel, a total of three different layouts of the cooling channel are designed, in which " $\otimes$ " and " $\odot$ "denote the inflow or outflow of fluid along the vertical direction of the paper surface, respectively.

Figure 6 shows the variation curve of the average temperature of the battery core axis in the case of each channel layout when the battery module is discharged at 4 $\mathrm{C}$ at the inlet velocity of $0.795 \times 10^{-2} \mathrm{~m} \cdot \mathrm{s}^{-1}$ in each cooling channel.

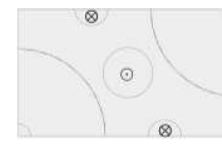

Case 1

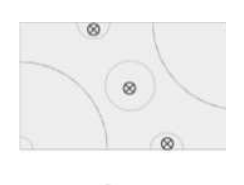

Case 2

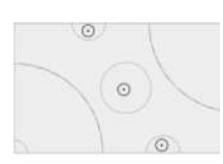

Case 3
Figure 5 The effect of flow direction

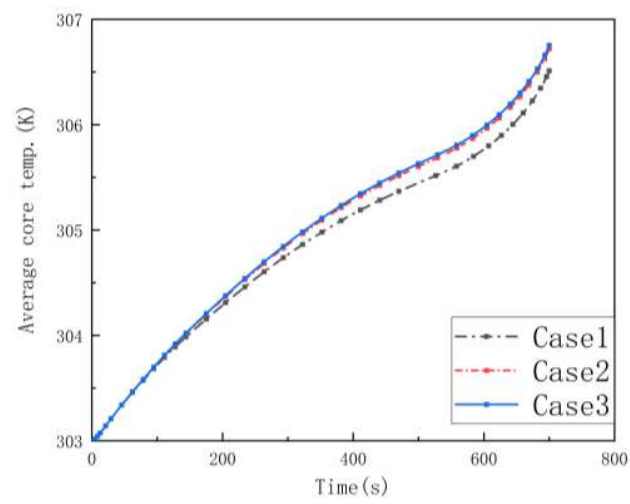

Figure 6 The average temperature variation along battery core axis

What can be noticed from Fig. 6 is that the average temperature of the central axis increases with time. The reason is that the "fast-slow-fast" trend of temperature variation is mainly attributed to the battery internal resistance [6]. In Fig.6, the temperature rise of all cases is within $4 \mathrm{~K}$, the average temperature rise of case 1 is the lowest, and the difference between case 2 and case 3 is within $0.2 \mathrm{~K}$.

Figure 7 shows the thermal profile of the battery and the temperature distribution on the surface of the BTM system at $700 \mathrm{~s}$. It can be seen that the battery temperature gradually increases along the axial direction and reaches the highest temperature at the core axis of the battery. In Case 1 of staggered cooling channel layout, the average temperature of the battery is low and the temperature distribution uniformity is higher.

In a word, the staggered flow channel arrangement has more advantages in controlling the heat production and thermal uniformity of the battery. The cooling channel 
arrangement of case 1 has better cooling performance for this BTM system.

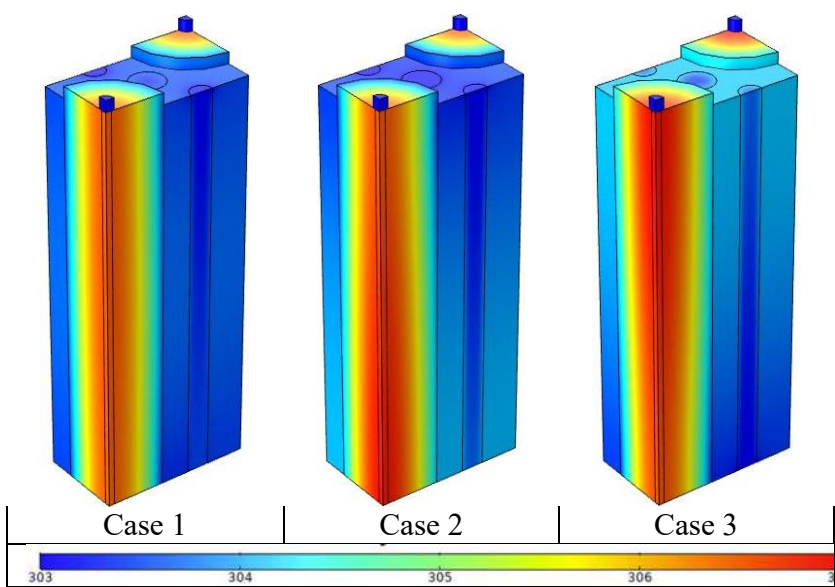

Figure 7 Thermal profile of battery module and surface temperature distribution of BTM system

\subsection{The effect of cooling channel size}

In this section, three different cooling channel diameters were initially designed, and the influence of different cooling channel sizes on heat distribution is preliminary studied. Figure 8 shows the cooling channel overlooking the cooling sleeve, where $d_{1} \sim d_{3}$ denotes the diameter of three adjacent cooling channels



Figure 8 The physical model of cooling channel

For the case of the battery module discharges at $4 \mathrm{C}$ rate, the inlet flow rate $0.795 \times 10^{-2} \mathrm{~m} \cdot \mathrm{s}^{-1}$, and the staggered flow arrangement of the cooling channel, the result of cooling channel size on battery temperature at axis center is depicted in Figure 9. Figure 10 shows the thermal profile of the battery module and the surface temperature distribution of the BTM system at each cooling channel size at 700s. When the diameter of the cooling channel $d_{1}$ and $d_{3}$ increases from $1.5 \mathrm{~mm}$ to $3.0 \mathrm{~mm}$ and $d_{2}$ increases from $2.0 \mathrm{~mm}$ to $4.5 \mathrm{~mm}$, the size of the cooling channel has the greatest impact on the temperature rise of the core axis of the 21700 battery, and the maximum temperature difference between all design sizes is within $0.5 \mathrm{~K}$.

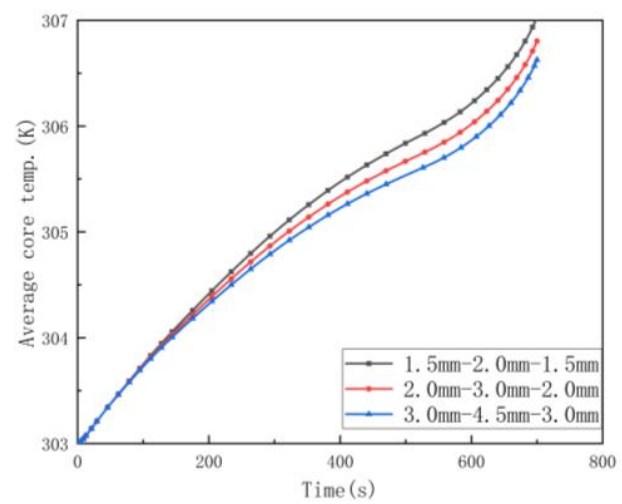

Figure 9 The result of cooling channel size on temperature curve of battery

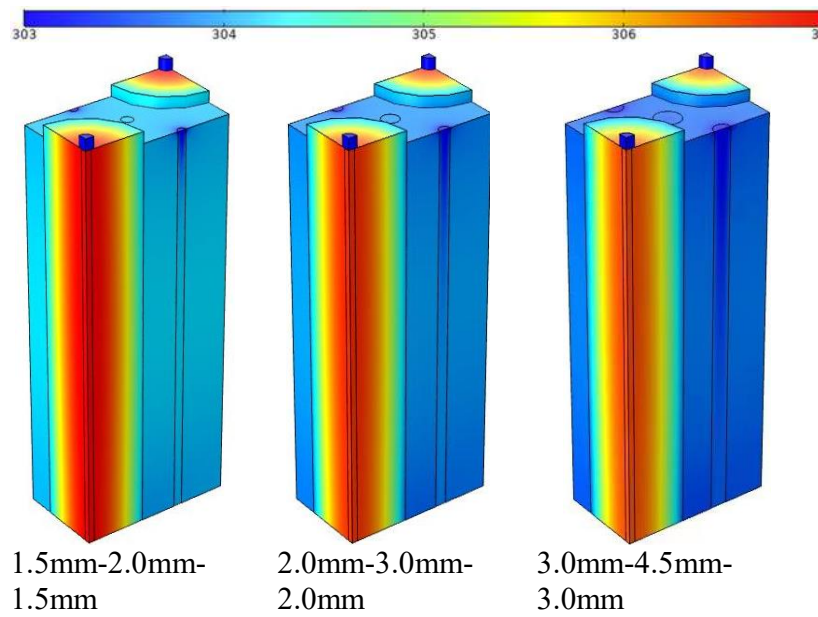

Figure 10 Thermal profile of battery modules with different cooling channel sizes and surface temperature distribution of BTM system

From the above results, expanding the diameter of cooling channel can enhance the cooling effect of BTM system to a certain extent. The next section will study the influence of cooling channel diameter $\left(d_{1}, d_{2}\right)$, he cooling jacket height $(\mathrm{h})$ and inlet velocity $\left(v_{0}\right)$ on heat dissipation of BTM system, and obtain the best design results within the acceptable parameter range. Using the Kriging method, in order to construct an approximate model to optimize the BTM system and improve the optimization efficiency and the optimization design, for these factors that based on multiobjective optimization design work.

\section{Approximate model and multiobjective optimization of BTM system}

The overall flow chart of this study is shown on Figure 11. Use COMSOL for numerical calculations. First of all, 
completing the geometric design of the BTM system, and the initial conditions are taken for numerical calculation. Secondly, to obtain the corresponding output target through the generated sample points, the DOE method is considered to get 30 sample points. The Kriging method is used to construct an approximate model, considering multidirection and multi-optimization target optimization schemes, including design parameters, target parameters and constraints. Finally, using the NSGA-II method [23, 24] in order to operate the optimization model to obtain a Pareto surface considering the average cell temperature (ACT) and the maximum temperature difference (MTD). Finally, compare the performance difference between the bestoptimized design and the initial unoptimized design, and select the best design.

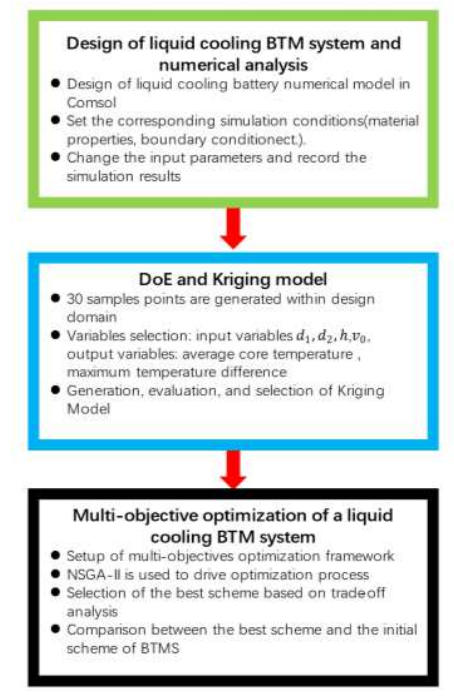

Figure 11 Flowchart of the research framework

\subsection{Approximate model}

The Kriging method is constructed to establish a highdimensional approximate model. It can effectively improve the numerical simulation calculation cost while obtaining numerical calculation accuracy. Kriging is an approximate model method that is generally used to approximate a set of input and output variables.

Figure 12 shows the flowchart of the establishment for Kriging model. In order to perform numerical simulation, take the numeral calculation on sampling points, the DoE sampling strategy is a method which is used to choose a series of sampling points. Construct the basis function, input the calculation results of the above sample points into the kriging model, and finally obtain the fitting curve of the first-order term and the fitting surface of the second-order term. After that, check the accuracy of the Kriging model and determine whether to use the model.



Figure 12 Flowchart of Kriging model

\subsection{Evaluation of the approximate model}

The range of influencing parameters needs to consider the structural stability and practicality of the proposed BTM system, which are shown in Table 1.

Table 1 The design variables and corresponding variation range

\begin{tabular}{llllll}
\hline The Variables & Symbol & $\begin{array}{l}\text { The } \\
\text { Unit }\end{array}$ & $\begin{array}{l}\text { The } \\
\text { Lower } \\
\text { limit }\end{array}$ & $\begin{array}{l}\text { The } \\
\text { Upper } \\
\text { limit }\end{array}$ & $\begin{array}{l}\text { The } \\
\text { Original } \\
\text { value }\end{array}$ \\
\hline Diameter of channel 1 & $d_{1}$ & $\mathrm{~mm}$ & 2.2 & 6.8 & 1.5 \\
Diameter of channel 2 & $d_{2}$ & $\mathrm{~mm}$ & 3.0 & 6.6 & 2 \\
Height of cooling jacket & $\mathrm{h}$ & $\mathrm{mm}$ & 65.0 & 70.0 & 68 \\
Inlet velocity & $v_{0}$ & $\mathrm{~m} / \mathrm{s}$ & 0.001 & 0.0725 & 0.005 \\
\hline
\end{tabular}

The established approximate model is based on the Kriging method [25]. The research is based on the numerical simulation calculation when the battery pack is discharged at a $4 \mathrm{C}$ discharge rate. This approach is to ensure that the proposed BTM system can meet the battery thermal energy dissipation requirements. In order to verify the approximate model of the BTM system, select and calculate 8 groups of sample points related to 4 factors in sequence. To evaluate the accuracy of the fit, $\mathrm{R}$-square $\left(\mathrm{R}^{2}\right)$ and root mean square (RMSE) are introduced. The calculation formula for the accuracy of the constructed approximate model is as follows:

$$
\begin{aligned}
& R^{2}=1-\frac{\sum_{\mathrm{i}=1}^{m}\left(f\left(x^{i}\right)-\hat{f}\left(x^{i}\right)\right)^{2}}{\sum_{\mathrm{i}=1}^{\mathrm{m}}\left(f\left(x^{i}\right)-\bar{f}\right)^{2}} \\
& R M S E=\frac{\sum_{i=1}^{m}\left(f\left(x^{i}\right)-\hat{f}\left(x^{i}\right)\right)^{2}}{m \cdot S T D}
\end{aligned}
$$


The established approximate model is based on the Kriging method [25]. The research is based on the numerical simulation calculation when the battery pack is discharged at a $4 \mathrm{C}$ discharge rate. This approach is to ensure that the proposed BTM system can meet the battery thermal energy dissipation requirements. In order to verify the approximate model of the BTM system, select and calculate 8 groups of sample points related to 4 factors in sequence. To evaluate the accuracy of the fit, R-square $\left(\mathrm{R}^{2}\right)$ and root mean square (RMSE) are introduced. The calculation formula for the accuracy of the constructed approximate model is as follows:

Table 2 Accuracy of Kriging models

\begin{tabular}{lll}
\hline Output & $R M S E$ & $R$-square \\
\hline MTD & 0.09788 & 0.91318 \\
ACT & 0.1196 & 0.90002 \\
\hline
\end{tabular}

The accuracy of Kriging model can be noticed from Table 2 is sufficient. In the next section, the MTD and ACT approximate models for the multiobjective optimization scheme of BTM systems are introduced.

\subsection{Multiobjective design optimization scheme}

This liquid cooling BTM system use multiobjective design optimization (MODO) of the has two optimization objectives, namely the minimization of MTD and ACT. On the premise of satisfying the requirements of battery thermal energy and reducing the risk of heat runaway transmission, improving the performance of the battery thermal management system, the average core temperature and the maximum temperature difference of the battery are the optimization goals. Four highly sensitive influencing factors are selected as the input parameters of the optimized design, including cooling channel diameter $\left(d_{1}, d_{2}\right)$, the height of cooling jacket $(\mathrm{h})$ and cooling channel inlet velocity $\left(v_{0}\right)$. The constraints of the proposed optimization model can be expressed in the following form:

$$
\left\{\begin{array}{c}
\text { variables } \overrightarrow{\mathrm{x}}=\left[\mathrm{d}_{1}, \mathrm{~d}_{2}, \mathrm{~h}, \mathrm{v}_{0}\right] \\
\text { MTD }=\mathrm{f}_{\mathrm{MTD}}(\overrightarrow{\mathrm{x}}) \\
\text { ARC }=\mathrm{f}_{\text {ATC }}(\overrightarrow{\mathrm{x}}) \\
\text { objectives find } \overrightarrow{\mathrm{x}}=\left[\mathrm{d}_{1}, \mathrm{~d}_{2}, \mathrm{~h}, \mathrm{v}_{0}\right] \\
\text { min MTD } \\
\text { min ACT } \\
\text { constraints } 2.2 \leq \mathrm{d}_{1} \leq 6.8 \\
3.0 \leq \mathrm{d}_{2} \leq 6.6 \\
65.0 \leq \mathrm{h} \leq 70.0 \\
0.001 \leq \mathrm{v}_{0} \leq 0.0725
\end{array}\right.
$$

\section{Results and discussions}

\subsection{Average core temperature (ACT)}

$\mathrm{ACT}$ is the average temperature of the battery core when the battery reaches the cut-off voltage during the discharge process. Therefore, ACT can be used to reflect the cooling efficiency of BTM system. As shown in Figure 13 (a d), a single variable has an effect on the average core temperature, while Figure 14(e $\mathrm{g})$ shows the influence of two parameters on the average core temperature.





Figure 13 The prediction of the average core temperature of BTM system (first-order terms)

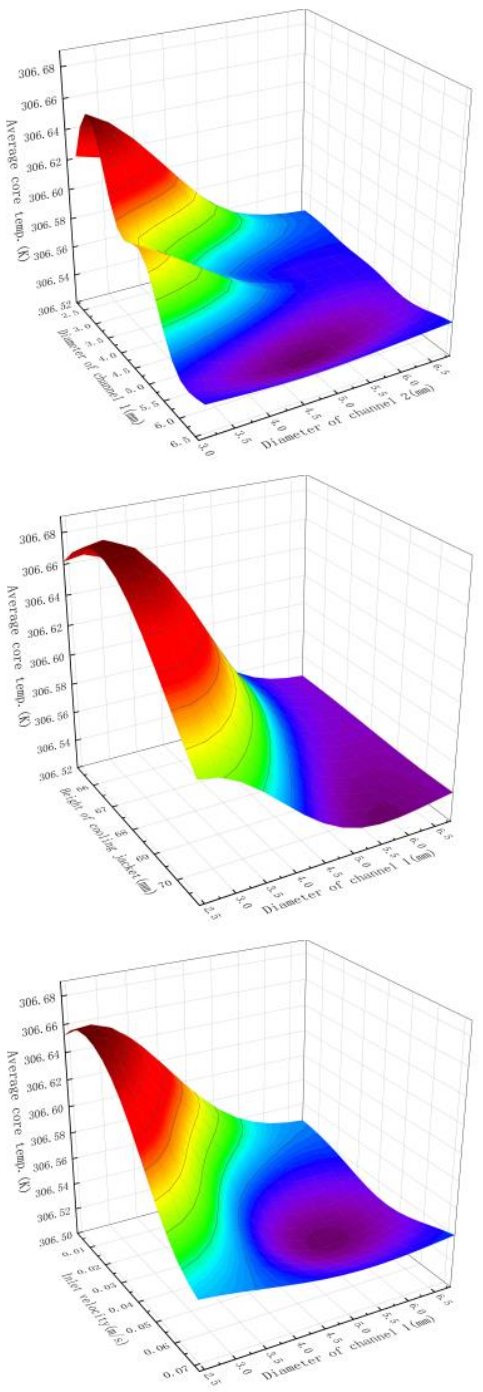

Figure 14 The prediction of the average core temperature of BTM system (second-order terms)

As can be seen from Figure 13(a), (b) and Figure 14 (e), ACT decreases with the increase of cooling channel diameter, with different trends, and, at the same time, as $d_{1}$ and $d_{2}$ increase, the downward trend of ACT gradually decreases. Figure 13(c) and Figure 14(f) show that increasing the height of the cooling jacket can reduce the ACT, but the trend is slight. And according to Figure 13(d) and Figure 14(g), expanding the inlet coolant flow velocity can make the ACT lower. Therefore, to improve the thermal energy dissipation efficiency, increasing the diameter of the cooling channel and increasing the flow velocity can be prioritized.

\subsection{Maximum temperature difference (MTD)}

MTD is the parameter that represent the uniformity of the battery surface heat distribution. In contrast to ACT, the influence of geometrical parameters of cooling passage on MTD is not significant, and Figure 15 (c), Figure 16 (f) show that MTD decreases with the increase of cooling jacket height. Figure 15(d) and Figure 16(g) show that MTD increases first and then decreases with the increase of coolant inlet velocity. Therefore, specific inlet velocity can be selected to enhance the cooling efficiency of BTM system.



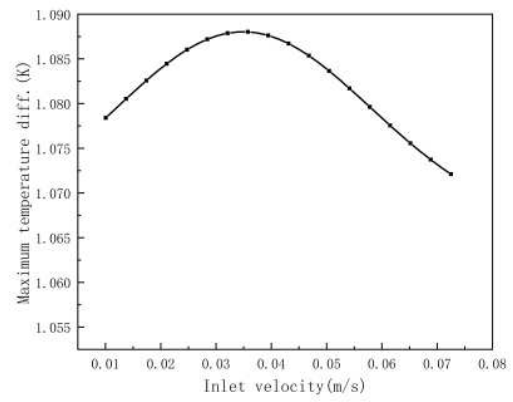

Figure 15 The prediction of the maximum temperature difference of BTM system (first-order terms)

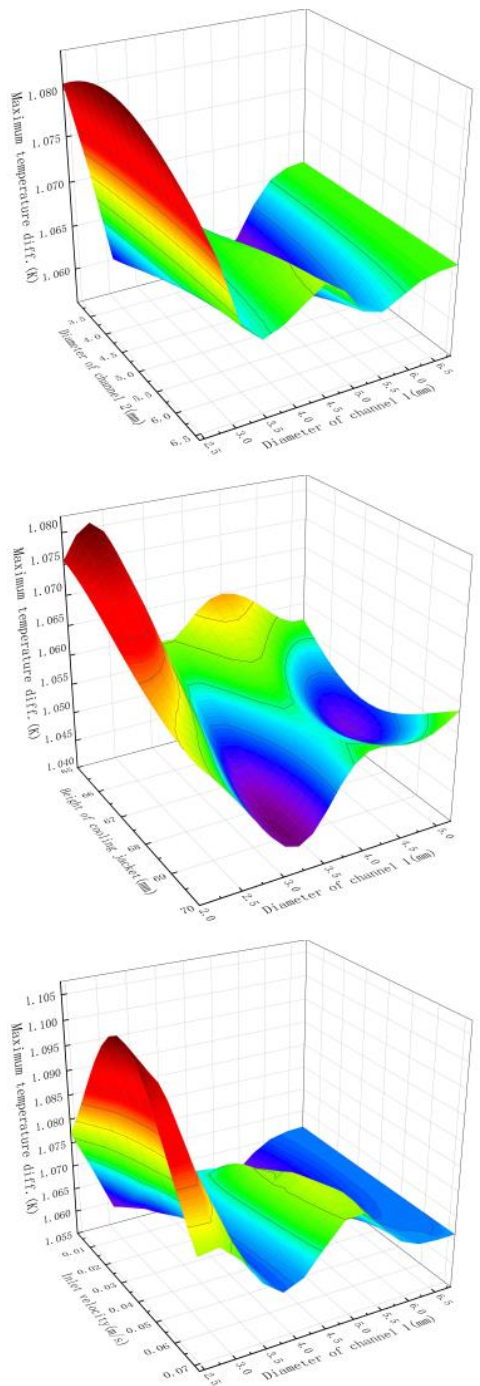

Figure 16 The prediction of the maximum temperature difference of BTM system (second-order terms)

And we can conclude, the expand of the diameter of the cooling channel $\left(d_{1}, d_{2}\right)$ can significantly reduce ACT and the height of the cooling jacket can reduce MTD. The diameter of channel $2\left(d_{2}\right)$ almost has no effect on MTD change. Thus, Larger cooling channel diameter and in order to improve the overall performance of the system, we can choose to increase the inlet coolant flow rate.

\subsection{Multiobjective optimization result}

When finish establishing the MOPSO framework, the NSGA-II algorithm is used to construct the optimization work. For the setting of NSGA-II, the population size, crossover probability, and the number of generations are 12 , 0.9 , and 20, respectively. The Pareto solution set based on the MOPSO algorithm is shown on Figure 17, and Pareto solution set Front arc AB is Pareto optimal solution set.

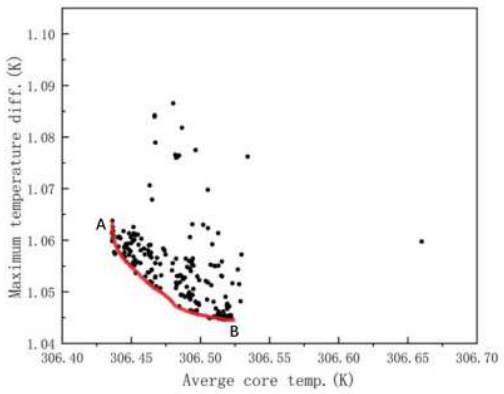

Figure 17 Pareto solution set of average core temperature and maximum temperature difference

According to the result of Pareto solution set and Pareto optimal solution set, the inability to fully achieve good heat dissipation as a whole may result in higher battery temperature differences, with an overall negative correlation between the average core temperature and the maximum temperature difference. Within the average core temperature range, the maximum temperature difference stabilizes in the average core temperature range of $306.45 \mathrm{~K}$ to $306.55 \mathrm{~K}$, but increases sharply when the average core temperature is below $306.45 \mathrm{~K}$. Table 3 shows the parameters of optimized and the original design. When the battery is discharged at $4 \mathrm{C}$ discharge rate, the optimized average core temperature battery is $306.44 \mathrm{~K}$, this result is lower than the original design. Compared with the previous design, through the maximum temperature difference is little larger, it remains within an acceptable range.

Table 3 Original parameters and optimized parameter values

\begin{tabular}{ccll}
\hline & $\begin{array}{c}\text { The Design } \\
\text { variables }\end{array}$ & \multicolumn{1}{c}{$\begin{array}{c}\text { Original } \\
\text { design }\end{array}$} & $\begin{array}{c}\text { Optimized } \\
\text { design }\end{array}$ \\
\hline The Input & $\mathrm{d}_{1}(\mathrm{~mm})$ & 1.5 & 4.3 \\
parameters & $\mathrm{d}_{2}(\mathrm{~mm})$ & 2.0 & 4.9 \\
& $\mathrm{~h}(\mathrm{~mm})$ & 68.00 & 68.19 \\
\hline
\end{tabular}




\begin{tabular}{ccll}
\hline & $\mathrm{v}_{0}(\mathrm{~m} / \mathrm{s})$ & 0.005 & 0.045265 \\
\hline The Output & $\begin{array}{c}\text { average core } \\
\text { temperature }(\mathrm{K})\end{array}$ & 307.03 & 306.44 \\
parameters & $\begin{array}{c}\text { maximum } \\
\text { temperature } \\
\text { difference }(\mathrm{K})\end{array}$ & 1.03 & 1.0605 \\
& & & \\
\hline
\end{tabular}

\section{Conclusion}

A new type of BTM system for electric vehicles based on liquid cooling is developed to enhance heat transfer in the battery packs. The Kriging method is used to establish an approximate numerical model of the heat transfer in the BTM system. There are four sensitive factors that affect the average temperature of battery core and maximum temperature difference considered by the approximate model. After constructing the approximate model, the MOPSO method is used to optimize the design of the BTM system, and the performance conditions of the optimized system are tested under $4 \mathrm{C}$ discharge rate. The results shows that this new battery pack BTM system can obtain a lower rise of average temperature of battery core and more uniform temperature distribution by arranging the cooling channels in the staggered flow direction, the approximate model of the Kriging method can significantly reduce the cost of numerical simulation calculations of the optimal design under the condition of high calculation accuracy. Compared with the BTM system under the original design parameters, the optimized BTM system has the greatest thermal energy dissipation performance and better cooling uniformity. The average core temperature is $306.44 \mathrm{~K}$, and the difference of battery surface temperature is smaller. As the optimized BTM system has excellent heat dissipation performance, the risk of thermal runaway propagation will be reduced even under thermal runaway conditions.

\section{Declaration}

\section{Acknowledgements}

The authors sincerely thanks to Professor Jing Ma of Chang'an University for her critical discussion and reading during manuscript preparation.

\section{Funding}

Supported by the National Natural Science Foundation of China (Granted Nos. 51976173, 51976014), Jiangsu Provincial Natural Science Foundation of China (Granted No. BK20201204), and Basic Research Program of Taicang (Granted No. TC2019JC01).

\section{Availability of data and materials}

The datasets supporting the conclusions of this article are included within the article.

\section{Authors' contributions}

The author' contributions are as follows: Yasong Sun was in charge of the whole trial; Yasong Sun wrote the manuscript; Ruihuai Bai assisted with sampling and laboratory analyses.

\section{Competing interests}

The authors declare no competing financial interests.

\section{Consent for publication \\ Not applicable}

\section{Ethics approval and consent to participate}

Not applicable

\section{References}

[1] T Bandhauer, S Garimella, TF Fuller. A critical review of thermal issues in lithium-ion batteries. Journal of the Electrochemical Society, 2011, 158(3): R1.

[2] L Sheng, L Su, H Zhang, et al. Numerical investigation on a lithiumion battery thermal management utilizing a serpentine-channel liquid cooling plate exchanger. International Journal of Heat and Mass Transfer, 2019, 141: 658-668.

[3] Q Wang, B Jiang, B Li, et al. A critical review of thermal management models and solutions of lithium-ion batteries for the development of pure electric vehicles. Renewable and Sustainable Energy Reviews, 2016, 64: 106-128.

[4] E Jiaqiang, Y Meng, JW Chen, et al. Effects of the different aircooling strategies on cooling performance of a lithium-ion battery module with baffle. Applied Thermal Engineering, 2018, 144: 231241.

[5] S Landini, J Leworthy, TS O'Donovan. A review of phase change materials for the thermal management and isothermalisation of lithium-ion Cells. Journal of Energy Storage, 2019, 25: 10087.

[6] H Yang, H Zhang, S Yang, et al. Numerical analysis and experimental visualization of phase change material melting process for thermal management of cylindrical power battery. Applied Thermal Engineering, 2018, 128: 489-499.

[7] L Jiang, HY Zhang, JW Li, et al. Thermal performance of a cylindrical battery module impregnated with PCM composite based on thermoelectric cooling. Energy, 2019, 188: 116048.

[8] J. Chen, S. Kang, E Jiaqiang, et al. Effects of different phase change material thermal management strategies on the cooling performance of the power lithium-ion batteries: A review. Journal of Power Sources, 2019, 442: 227228.

[9] E Jiaqiang, Y Zeng, Y Jin, et al. Heat dissipation investigation of the power lithium-ion battery module based on orthogonal experiment design and fuzzy grey relation analysis. Energy, 2020, 211: 118596.

[10]XH Zhao, E Jiaqiang, G Wu, et al. A review of studies using graphenes in energy conversion, energy storage and heat transfer development. Energy Conversion and Management, 2019, 184: 581- 
599

[11]S Kohn, G Berdichevsky, B Hewett. Tunable frangible battery pack system. US Patent No. 7.923.144, April 12, 2011.

[12]JT Zhao, ZH Rao, YM Li. Thermal performance of mini-channel liquid cooled cylinder-based battery thermal management for cylindrical lithium-ion power battery. Energy Conversion and Management, 2015, 103: 157-165.

[13]ZH Rao, Z Qian, Y Kuang, et al. Thermal performance of liquid cooling based thermal management system for cylindrical lithium-ion battery module with variable contact surface. Applied Thermal Engineering, 2017, 123: 1514-1522.

[14]L Sheng, HY Zhang, L Su, et al. Effect analysis on thermal profile management of a cylindrical lithium-ion battery utilizing a cellular liquid cooling jacket. Energy, 2021, 220: 119725.

[15]SA Hallaj, H Maleki, JS Hong, et al. Thermal modeling and design considerations of lithium-ion batteries. Journal of Power Sources, 1999, 83: 1-8.

[16]M Al-Zareer, I Dincer, MA Rosen. A review of novel thermal management systems for batteries. International Journal of Energy Research, 2018, 42: 3182-3205.

[17]ZH Rao, QC Wang, CL Huang. Investigation of the thermal performance of phase change material/mini-channel coupled battery thermal management system. Applied energy, 2016, 164: 659-669.

[18]C Multiphysics. Introduction to COMSOL Multiphysics. COMSOL Multiphysics, Burlington, MA, February 9, 2018.

[19]M Doyle, J Newman, AS Gozdz, et al. Comparison of modeling predictions with experimental data from plastic lithium-ion cells. Journal of the Electrochemical Society, 1996, 143(6): 1890.

[20]B Koo, P Goli, AV Sumant, et al. Toward lithium-ion batteries with enhanced thermal conductivity. ACS Nano, 2014, 8(7): 7202-7207.
[21]M Doyle, TF Fuller, J Newman. Modeling of galvanostatic charge and discharge of the lithium/polymer/insertion cell. Journal of the Electrochemical Society, 1993, 140(6): 1526.

[22]TF Fuller, M Doyle, J Newman. Simulation and optimization of the dual lithium-ion insertion cell. Journal of the Electrochemical Society, 1994, 141(1): 1.

[23]KK Alireza, H Mohammadi. Multiobjective optimization in pavement management system using NSGA-II method. Journal of Transportation Engineering, Part B: Pavements, 2018, 144(2): 04018016.

[24]S Ramesh, S Kannan, S Baskar. Application of modified NSGA-II algorithm to multiobjective reactive power planning. Applied Soft Computing, 2012, 12(2): 741-753.

[25]I Kaymaz. Application of kriging method to structural reliability problems. Structural Safety, 2005, 27(2): 133-151.

\section{Biographical notes}

Ya-Song Sun, born in 1986, is currently an associate professor at Northwestern Polytechnical University, China. He received his Ph. D. degree on mechanical engineering in Northwestern Polytechnical University, China, in 2011.

Tel: +86-29-88431112; E-mail: yssun@nwpu.edu.cn

Rui-Huai Bai, born in 1997, is currently a master candidate at Key Laboratory of Internal Aerodynamics in Aero-Engine, Northwestern Polytechnical University, China.

E-mail: 598295322@qq.com 